\title{
Infarto agudo al miocardio secundario a disección coronaria espontánea biarterial: Reporte de un caso y revisión de literatura.
}

\author{
Mathias Schwarze ${ }^{a}$, Bastián Abarca ${ }^{a}$, Rodrigo Contreras ${ }^{a}$, Pablo Rodríguez $^{a}$, Iván Roa $^{a}$, Hermann Schwarze $^{1}$. \\ 1. Cardiólogo, Clínica Integral Red Salud, Rancagua, Chile. \\ a. Interno de Medicina, Universidad San Sebastián, Santiago, Chile. \\ Financing: There was no external funding.
}

La disección coronaria espontánea ha surgido como una causa interesante y poco reconocida de síndrome coronario agudo no aterosclerótico. Ocurre más frecuentemente en mujeres jóvenes, donde de forma importante puede asociarse a anomalías arteriales no coronarias. La clave para un tratamiento oportuno es el diagnóstico precoz que impida su evolución a cuadros más severos. Se presenta el caso de una mujer de
36 años con un síndrome coronario agudo secundario a una disección coronaria espontánea bi-arterial que evolucionó de forma grave a una insuficiencia cardíaca aguda. El objetivo es informar un cuadro poco usual y proporcionar evidencia que respalde el cómo debe enfrentarse.

Palabras clave: intervención coronaria percutánea; infarto miocárdico; disección coronaria espontánea.

\section{Acute myocardial infarction due to spontaneous two vessel coronary artery dissection}

Spontaneous coronary dissection is an important cause of acute non-atherosclerotic coronary syndrome. It occurs more frequently in young women, often without significantly associated coronary arterial anomalies. Early diagnosis is a key to prompt treatment, in order to prevent severe complications. We report the case of a 36-year-old woman with an acute coro- nary syndrome secondary to spontaneous bi-arterial coronary dissection progressing to severe acute heart failure. A discussion of the management of this condition is included.

Keywords: Percutaneous coronary intervention, myocardial infarction, coronary artery dissection, spontaneous. 


\section{Introducción:}

La disección coronaria espontánea ha surgido como una causa importante y poco reconocida de síndrome coronario agudo no aterosclerótico, en particular entre mujeres jóvenes e individuos con pocos factores convencionales de riesgo cardiovascular ${ }^{1,2}$, que a la fecha se sigue diagnosticando de manera insuficiente ${ }^{3}$. Tiene una prevalencia estimada que varía de 0,1 a $1,1 \%$ en la mayoría de los estudios angiográficos4, siendo causante de alrededor del 35\% de los síndromes coronarios agudos en mujeres menores de 50 años y de $10 \% 10 \%$ en hombres ${ }^{5}$.

El mecanismo fisiopatológico es la rotura primaria de la túnica íntima, generando un sangrado que desarrolla una luz falsa que se comunica con la luz verdadera. La expansión impulsada por presión de la luz falsa conduce a la propagación de la disección y la compresión adicional de esta sobre la luz verdadera ${ }^{4}$. Alternativamente, en caso de no existir un desgarro de la túnica íntima, es probable que la interrupción primaria de los "vasa vasorum" conduzca a un hematoma intramural, sin comunicación entre el lumen verdadero y el falso ${ }^{4}$. Cada uno de estos no tiene una etiología claramente establecida; sin embargo, se puede asociar a condiciones que predispongan su ocurrencia.

Esto recalca que aún no existan factores que predigan la historia natural ni el pronóstico de la enfermedad. La práctica actual de manejo se basa, principalmente, en datos retrospectivos, faltando series de casos y directrices que definan protocolos de manejo claro ${ }^{6}$. Este es un nuevo reporte de caso de un infarto agudo al miocardio extenso, secundario a disección coronaria espontánea y que evolucionó a insuficiencia cardíaca aguda.

\section{Presentación del caso}

Mujer de 36 años de edad, con antecedentes mórbidos de obesidad, dislipidemia y tabaquismo crónico, sin hipertensión arterial conocida. Consulta por dolor precordial de inicio súbito, de 90 minutos de duración, muy intenso, con escala visual análoga 10/10, que se irradia a la extremidad superior izquierda; además, disnea, náuseas, palidez y diaforesis. Un ECG mostró sinusal con supradesnivel del segmento ST de V1 a V6. Se inició protocolo

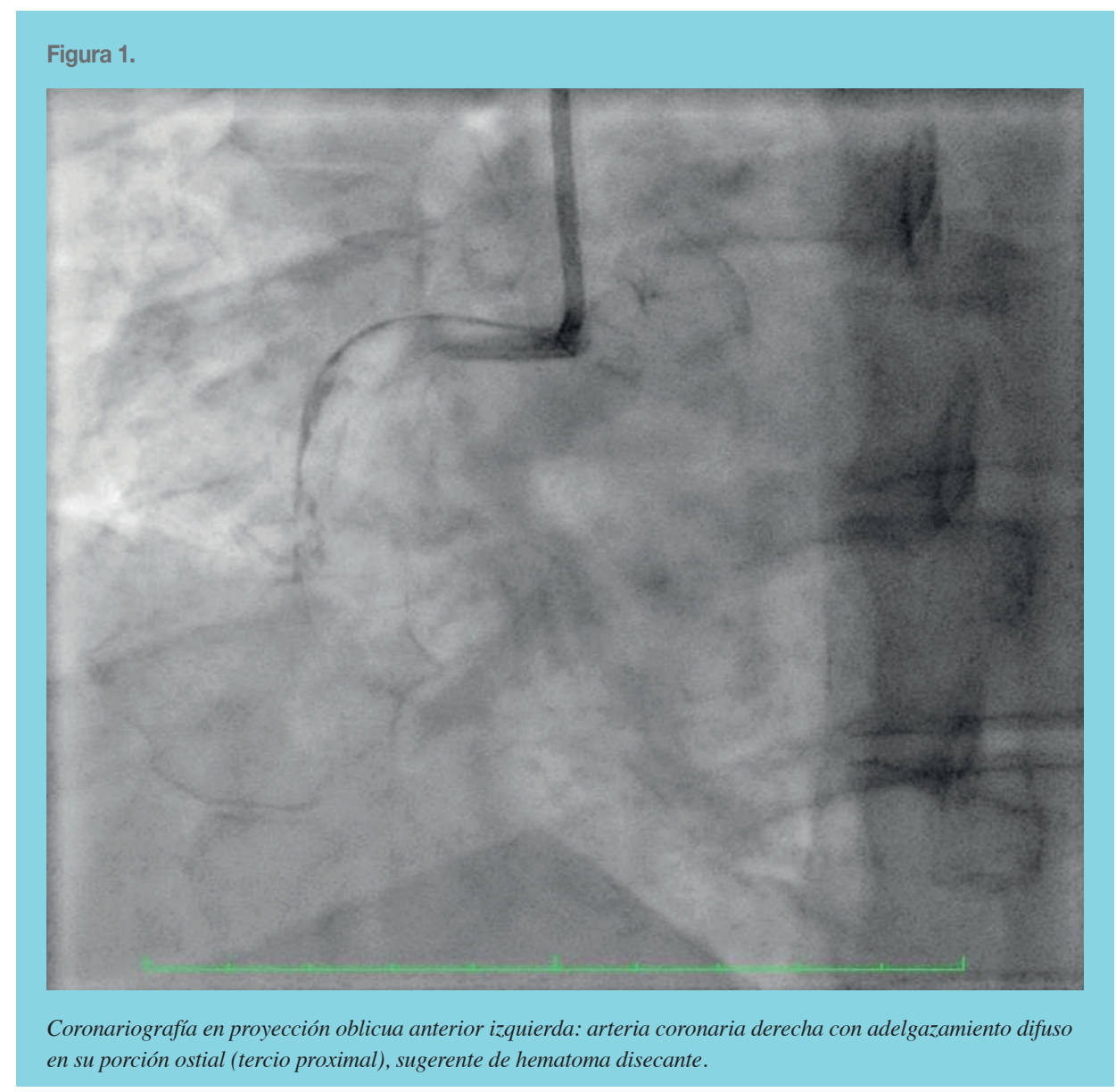





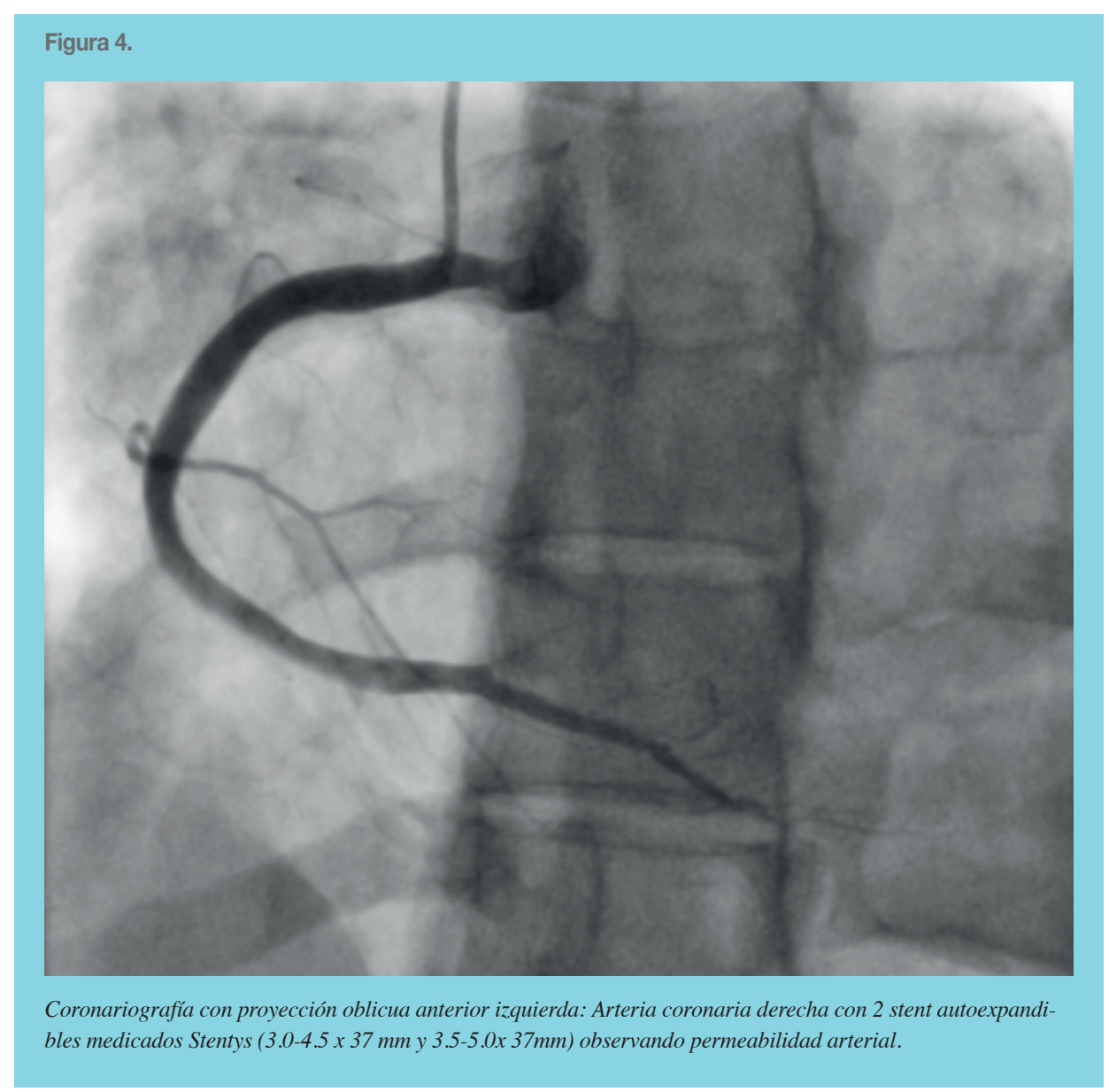

de fibrinolisis con tenecteplase, que tuvo efecto clínico inmediato disminuyendo la intensidad del dolor a una escala visual análoga 7/10. Fue trasladada a centro hospitalario para completar estudio y manejo hemodinámico.

A su ingreso a la unidad de emergencia destaca una presión arterial de 202/124 mmHg, frecuencia cardíaca de 76 latidos por minuto y saturación de oxígeno de $99 \%$ ambiental, sin anormalidades en el examen físico. El primer electrocardiograma muestra un ritmo sinusal con supradesnivel del segmento ST en DII, DIII, aVF y de V2 a V6; el segundo electrocardiograma muestra un patrón evolutivo con aparición de una onda Q patológica en DII, DIII y aVF; el tercero mantiene dicho patrón evolutivo, ahora con onda Q patológica de V3 a V5. Los exámenes de laboratorio muestran una CK-TOTAL $6430 \mathrm{U} / \mathrm{L}$ (valor normal de referencia $<170 \mathrm{U} / \mathrm{L}$ ), una CK-MB $735 \mathrm{U} / \mathrm{L}$ (valor normal de referencia $0-25 \mathrm{U} / \mathrm{L}$ ) y una Troponina-I $>100 \mu \mathrm{g} / \mathrm{dl}$ (valor normal de referencia $<0,50 \mu \mathrm{g} / \mathrm{dl}$ ). Se inicia tratamiento farmacológico con enoxaparina, ácido acetil-salicílico, clopidogrel, atorvastatina e infusión continua de nitroglicerina, además de traslado a unidad de cuidados intensivos y estudio hemodinámico, cuya coronariografía revela una disección coronaria espontánea de la arteria coronaria derecha ostial y de la arteria descendente anterior izquierda en su porción media (Figura 1). Se evidenció una disfunción sistólica severa del ventrículo izquierdo con una fracción de eyección estimada de $25 \%$. Se completa estudio con Angio TAC de tórax, que descarta un síndrome aórtico.

Al sexto día de hospitalización evoluciona con nuevo episodio de dolor torácico opresivo, cuyos exámenes de laboratorio destacan Troponinas T Ultrasensible $5440 \mathrm{pg} /$ $\mathrm{mL}$ (valor normal de referencia $<14 \mathrm{pg} / \mathrm{ml}$ ), CK-Total 4437 U/L y CK-MB 360 U/L. Se le realiza un nuevo estudio hemodinámico con una coronariografía que muestra una arteria coronaria derecha con oclusión distal y una arteria descendente anterior izquierda ocluida en tercio medio (secundario a la disección), manejadas con la instalación de stents autoexpandibles medicados "Stentys ${ }^{\circledR}$ (3.0-4.5 x 37mm y $3.5-5.0$ x $37 \mathrm{~mm})$ " en arteria coronaria 
derecha (Figuras 3 y 4).

Los siguientes 7 días evoluciona de manera estable, siendo incorporada al programa de trasplante cardiovascular y dada de alta con diagnósticos de emergencia hipertensiva secundaria a infarto agudo al miocardio con supradesnivel del segmento ST, disección coronaria espontánea con oclusión secundaria de las arterias coronarias derecha y descendente anterior izquierda (manejada con 2 stent medicados) e insuficiencia cardíaca por disfunción sistólica moderada.

En el control ambulatorio un estudio de perfusión miocárdica MIBI dipiridamol mostró una cavidad ventricular izquierda dilatada con volumen de fin de diástole de 152 $\mathrm{ml}$ en reposo y $155 \mathrm{ml}$ post-estrés. En imágenes de estrés se observa un extenso defecto anteroapical con extensión septal medioapical, inferoapical y lateroapical, que compromete el $45 \%$ del ventrículo izquierdo, y un defecto inferobasal que compromete un $7 \%$ de la pared ventricular. Se observó aquinesia apical e hipoquinesia inferior. La función sistólica con una fracción de eyección del 36\% post-estrés y de $39 \%$ en reposo. Por tanto, dada esta mejoría de la función cardíaca, se retira como candidata a trasplante y se mantiene con controles por especialista.

\section{Discusión:}

La disección espontánea de la arteria coronaria como causa infrecuente de infarto agudo al miocardio se ha hecho más frecuente. La conciencia diagnóstica contemporánea y la disponibilidad de imágenes intracoronarias han permitido conocer su forma de manifestación, Sin embargo, hasta la fecha no se dispone de evidencia que respalde su tratamiento. Se asocia con el sexo femenino (81\% - 92\%), edad temprana (42 a 53 años), estrés emocional extremo o esfuerzo extremo $(12 \%-48 \%)$, embarazo $(18 \%)$ y la displasia fibromuscular ${ }^{7}$. Otros factores de riesgo menos influyentes son la hipertensión arterial $(27 \%-36 \%)$, diabetes mellitus $(0,9 \%-4,6 \%)$, tabaquismo $(0,6 \%-10 \%)$, consumo de cocaína, anticonceptivos orales, vasoespasmo coronario, vasculitis y síndromes genéticos (5\%) como el síndrome de Marfán, Ehlers-Danlos y Loeys-Dietz ${ }^{8}$.

La presentación más común es el síndrome coronario agudo, variando desde angina inestable hasta infarto agudo de miocardio, incluso shock cardiogénico. Su detección clínica es baja $(0,1 \% \text { a } 0,28 \%)^{9}$, incluyendo otras formas menos frecuentes de presentación como la insuficiencia cardíaca $(2 \%-3,9 \%)$ y el taponamiento cardíaco ${ }^{8,9}$. El diagnóstico se confirma mediante angiografía coronaria, donde el $67 \%$ de los casos afecta a la arteria descendente anterior, el $19 \%$ a la arteria coronaria derecha, el 14\% a la arteria coronaria circunfleja y menos del $1 \%$ afecta al tronco coronario izquierdo ${ }^{10}$. Si bien la angiografía coronaria es el gold standard para el estudio de este cuadro, tiene algunas limitaciones que hace necesario complementar con otros estudios para reducir la incidencia de un diagnóstico erróneo.

Los manejos reportados se basan en 3 aspectos: En primer lugar, el tratamiento médico conservador que incluye antiagregantes plaquetarios, heparina, beta-bloqueadores e inhibidores de la enzima convertidora de angiotensina ${ }^{11}$. En segundo lugar, la implantación de stent4. En tercer lugar, el bypass de la arteria coronaria para pacientes con disección del tronco coronario izquierdo o lesiones complejas, especialmente, aquellos con inestabilidad hemodinámica $^{12}$.

En este contexto, la coronariografía permitió identificar en forma acertada la disección coronaria espontánea biarterial con un flap de disección no oclusivo (sin placas de ateromas visibles), por lo que no hubo necesidad de instalar stents. Sin embargo, evolucionó posteriormente con isquemia, requiriendo una segunda coronariografía donde se implantaron dos stents autoexpandibles para mantener el flap de disección permeable, logrando la estabilización del cuadro y una evolución satisfactoria a una disfunción sistólica moderada.

Este caso mostró características poco atribuibles a una disección coronaria espontánea, dado que sí presentó factores de riesgo cardiovasculares y no estaba en un período peri/post parto, lo cual dejaba la disección coronaria espontánea en segundo plano. Por ello, se inició un protocolo de manejo como si fuese un síndrome coronario agudo por oclusión coronaria trombótica, cuya trombolisis pudo aumentar el riesgo vital de la paciente y en estos casos queda contraindicada. Finalmente, el diagnóstico de disección coronaria espontánea biarterial representa una condición sumamente infrecuente y poco divulgada dentro estos cuadros, donde los reportes de afección de múltiples vasos son extremadamente escasos ${ }^{13}$.

\section{Conclusión:}

La disección coronaria espontánea se distingue cada vez más como una etiología importante del infarto agudo de miocardio y debe sospecharse en mujeres jóvenes o de mediana edad con clínica sugerente. El diagnóstico oportuno va a ser un elemento clave para ofrecer un cambio en el pronóstico de estos cuadros, basados en la terapia que proporcione la evidencia. 


\section{Referencias}

1. MAIN T, PRAKASH R, STAROVOYTOV A, SABBAGHAN A, AYMONG E, MANCINI G, et al. Characteristics of extension and de novo recurrent spontaneous coronary artery dissection. Eurointervention. 2017;13:e1454-e1459. DOI: 10.4244/EIJ-D-17-00264. https://www.ncbi.nlm.nih. gov/pubmed/28891472

2. HAYES S, KIM E, SAW J, ADLAM D, ARSLANIAN-ENGOREN C, ECONOMY K, et al. Spontaneous Coronary Artery Dissection: Current State of the Science: A Scientific Statement From the American Heart Association. Circulation. 2018;137:e523-e557. DOI: 10.1161/ CIR.0000000000000564. https://www.ahajournals.org/doi/ abs/10.1161/CIR.0000000000000564

3. SAW J, HUMPHRIES K, AYMONG E, SEDLAK T, PRAKASH R, STAROVOYTOV A, et at. Spontaneous Coronary Artery Dissection: Clinical Outcomes and Risk of Recurrence. J Am Coll Cardiol. 2017 Aug 29;70(9):1148-1158. DOI: $10.1016 /$ j.jacc.2017.06.053. https://www.ncbi.nlm.nih. gov/pubmed/28838364

4. ALFONSO F, BASTANTE T, GARCÍA-GUIMARAES M, POZO E, CUESTA J, RIVERO F, et al. Spontaneous coronary artery dissection: new insights into diagnosis and treatment. Coron Artery Dis. 2016 Dec;27(8):696-706. DOI: 10.1097/ MCA.0000000000000412. https://www.ncbi.nlm.nih.gov/ pubmed/27404579

5. AGRAWAL V, KIM E. Spontaneous Coronary Artery Dissection: Cardiac Manifestations of Vascular Disease. Prog Cardiovasc Dis. 2018 Mar - Apr;60(6):629-634. DOI: 10.1016/j.pcad.2018.04.001. https://www.ncbi.nlm.nih.gov/ pubmed/29630904

6. AHMED MA, HAMRA M, ALI M, ABDULLAH AS, ARNOUS S, KIERNAN TJ. Spontaneous coronary artery dissection, challenges of diagnosis and management. Future Cardiol. 2017 Nov;13(6):539-549. DOI: 10.2217/fca-2017-0050. https://www.ncbi.nlm.nih.gov/pubmed/29064286

7. TWEET MS, GULATI R, HAYES SN. Spontaneous Coronary Artery Dissection. Curr Cardiol Rep. 2016 Jul;18(7):60. DOI: 10.1007/s11886-016-0737-6. https://www.ncbi.nlm.nih.gov/ pubmed/27216840

8. TWEET M, KOK S, HAYES S. Spontaneous coronary artery dissection in women: What is known and what is yet to be understood. Clin Cardiol. 2018 Feb; 41(2): 203-210. DOI: 10.1002/clc.22909. https://www.ncbi.nlm.nih.gov/pmc/articles/PMC5953427/

9. YANG XQ, ZHU HY, WANG X, ZHAO HB, ZHANG W, $\mathrm{XIAO} \mathrm{M}$, et at. Spontaneous coronary artery dissection in a middle-aged woman with acute anterior myocardial infarction: A case report. Medicine (Baltimore). 2018 Aug;97(31):e11504. DOI: 10.1097/MD.0000000000011504. https://www.ncbi.nlm.nih.gov/pubmed/30075514

10. MENG P, XU C, YOU W, WU Z, XIE D, ZHANG H, et al. Spontaneous Coronary Artery Dissection as a Cause of Acute Myocardial Infarction in Young Female Population: A Single-center Study. Chin Med J (Engl). 2017 Jul 5;130(13):1534-1539. DOI: 10.4103/0366-6999.208245. https://www.ncbi.nlm.nih.gov/pubmed/28639567

11. ROGOWSKI S, MAEDER M, WEILENMANN D, HAAGER P, AMMANN P, ROHNER F, et al. Spontaneous Coronary Artery Dissection: Angiographic Follow-Up and LongTerm Clinical Outcome in a Predominantly Medically Treated Population. Catheter Cardiovasc Interv. 2017 Jan;89(1):5968. DOI: 10.1002/ccd.26383. https://www.ncbi.nlm.nih.gov/ pubmed/26708825

12. SAW J, MANCINI GB, HUMPHRIES K, FUNG A, BOONE R, STAROVOYTOV A, et al. Angiographic appearance of spontaneous coronary artery dissection with intramural hematoma proven on intracoronary imaging. Catheter Cardiovasc Interv 2016;87:E54-61. DOI: 10.1002/ccd.26022. https:// www.ncbi.nlm.nih.gov/pubmed/26198289

13. AKGUL A, GURSOY M, BAKUY V, KOMURCU G, CAGLAR I, GULMALIYEV J. Spontaneous triple coronary artery dissection. Ann Thorac Surg. 2013 Apr;95(4):1443-5. DOI: 10.1016/j.athoracsur.2012.08.080. https://www.ncbi. nlm.nih.gov/pubmed/23522210 